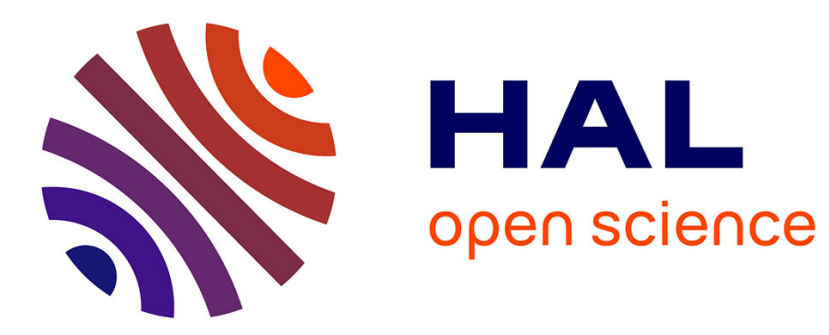

\title{
Coupling dynamic equations and satellite images for modelling ocean surface circulation
}

Dominique Béréziat, Isabelle Herlin

\section{To cite this version:}

Dominique Béréziat, Isabelle Herlin. Coupling dynamic equations and satellite images for modelling ocean surface circulation. Communications in Computer and Information Science, 2015, 550, pp.191205. 10.1007/978-3-319-25117-2_12 . hal-01245369

\section{HAL Id: hal-01245369 \\ https://hal.inria.fr/hal-01245369}

Submitted on 27 Apr 2016

HAL is a multi-disciplinary open access archive for the deposit and dissemination of scientific research documents, whether they are published or not. The documents may come from teaching and research institutions in France or abroad, or from public or private research centers.
L'archive ouverte pluridisciplinaire HAL, est destinée au dépôt et à la diffusion de documents scientifiques de niveau recherche, publiés ou non, émanant des établissements d'enseignement et de recherche français ou étrangers, des laboratoires publics ou privés. 


\title{
Coupling dynamic equations and satellite images for modelling ocean surface circulation
}

\author{
Dominique Béréziat ${ }^{1,2}$ and Isabelle Herlin ${ }^{3,4}$ \\ 1 Sorbonne Universités, UPMC Univ Paris 06, UMR 7606, LIP6, 4 place Jussieu, \\ F-75005, Paris, France \\ 2 CNRS, UMR 7606, LIP6, F-75005, Paris, France \\ 3 Inria, B.P. 105, 78153 Le Chesnay, France \\ 4 CEREA, joint laboratory ENPC - EDF R\&D, Université Paris-Est, Cité Descartes \\ Champs-sur-Marne, 77455 Marne la Vallée Cedex 2, France
}

\begin{abstract}
Satellite image sequences visualise the ocean surface and allow assessing its dynamics. Processing these data is then of major interest to get a better understanding of the observed processes. As demonstrated by state-of-the-art, image assimilation permits to retrieve surface motion, based on assumptions on the dynamics. In this paper, we demonstrate that a simple heuristics, such as the Lagrangian constancy of velocity, can be used and successfully replace the complex physical properties described by the Navier-Stokes equations for assessing surface circulation from satellite images. A data assimilation method is proposed that adds an acceleration term $\mathbf{a}(t)$ to this Lagrangian constancy equation, which summarises all physical processes other than advection. A cost function is designed that quantifies discrepancy between satellite data and model values. This cost function is minimised by the BFGS solver with a dual method of data assimilation. The result is the initial motion field and the acceleration terms $\mathbf{a}(t)$ on the whole temporal interval. These values $\mathbf{a}(t)$ model the forces, other than advection, that contribute to surface circulation. Our approach was tested on synthetic data and with Sea Surface Temperature images acquired on Black Sea. Results are quantified and compared to those of state-of-the-art methods.
\end{abstract}

Keywords: Dynamic model, Optical flow, Data Assimilation, Satellite image, Ocean circulation

\section{Introduction}

Satellite image sequences permit to visualise oceans' surface and their underlying dynamics with a high spatial resolution. Processing these images is then of major interest for a better understanding of the observed processes and the forecast of extreme events. As demonstrated by state-of-the-art, image assimilation allows to retrieve surface motion from image sequences, using heuristics on the dynamics [11 15]. Among those heuristics, the shallow water model [17] has been proven to be suitable for representing the surface circulation of closed seas, 
such as Black Sea [10. These shallow water equations have also been successfully used to estimate the upper layer circulation of Black Sea from Sea Surface Temperature (SST) images [516] with a data assimilation method.

In this paper, we propose to learn the surface dynamics from SST image acquisitions with a data assimilation method applied to an image model derived from the shallow water equations. In the shallow water model, surface circulation is characterised by the horizontal velocity, that is advected by itself and subject to geophysical forces such as Coriolis, Earth gravity and viscosity. The advection process is kept in the image model, but all other components are summarised in a global term, denoted a (letter a stands for acceleration) that is estimated by our approach. Adding this term to the advection is similar, from a mathematical point of view, to the weak data assimilation framework 2[12 16 18. A data assimilation technique is then designed to compute the solution: a cost function is constructed, whose control variables are the motion field at the first acquisition date and the acceleration values $\mathbf{a}(t)$, at all dates of the acquisition interval. The minimum of the cost function is obtained thanks to optimal control techniques [9] and it is computed with the BFGS solver [20].

Section 2 provides notations that are used in the remaining of the paper and the mathematical description of the proposed approach for modelling the dynamics of the ocean's upper layer. The data assimilation method is outlined in Section 3 It corresponds to a weak formulation, where the non advective terms are summarised as an additional term in the evolution equation. The implementation is shortly described in Section 4 in order to permit that readers apply the method by themselves. Validation on synthetic data and results on SST image sequences acquired over Black Sea by NOAA-AVHRR sensors are displayed and quantified in Section 5.

\section{Problem statement}

Image data are acquired on a bounded rectangle of $\mathbb{R}^{2}$, named $\Omega$, and on a temporal interval $[0, \mathrm{~T}]$. Let define $A=\Omega \times[0, \mathrm{~T}]$ the corresponding spacetime domain, on which the dynamics is modelled. A point $\mathbf{x} \in \Omega$ is defined as $\mathbf{x}=(x y)^{T}$ and the motion vector at point $\mathbf{x}$ and date $t \in[0, \mathrm{~T}]$ is written $\mathbf{w}(\mathbf{x}, t)=(u(\mathbf{x}, t) v(\mathbf{x}, t))^{T}$. At each date $t$, the motion field on the domain $\Omega$ is written as $\mathbf{w}(t) . N$ Sea Surface Temperature acquisitions are available at dates $t_{i}, i=1 \cdots N$. They are denoted $T\left(t_{i}\right)$ with pixels values $T\left(\mathbf{x}, t_{i}\right)$.

A state vector $\mathbf{X}$ is defined on $A$. It includes the two components $u$ and $v$ of the motion vector $\mathbf{w}(\mathbf{x}, t)$ and a pseudo-temperature value $T_{M}(\mathbf{x}, t)$, which has properties similar to those of the Sea Surface Temperature function:

$$
\mathbf{X}(\mathbf{x}, t)=\left(\mathbf{w}(\mathbf{x}, t)^{T} T_{M}(\mathbf{x}, t)\right)^{T}
$$

The index $M$ in $T_{M}$ reminds that this is a component of the $M$ odel state vector. At the end of the data assimilation process, the discrepancy between the pseudotemperature $T_{M}$ and the satellite acquisitions $T$ has to be small. 
The heuristics on dynamics, used in the paper, are derived from the shallow water equations that express the principles of mass and momentum conservation [17. Circulation of the upper ocean is represented by the $2 \mathrm{D}$ velocity $\mathbf{w}=(u v)^{T}$ and the thickness $h$ of the mixed layer. In our model, the pseudotemperature $T_{M}$ is transported by the motion field. This provides the following set of equations:

$$
\begin{aligned}
\frac{\partial u}{\partial t} & =-u \frac{\partial u}{\partial x}-v \frac{\partial u}{\partial y}+f v-g^{\prime} \frac{\partial \eta}{\partial x}+K_{\mathbf{w}} \Delta u \\
\frac{\partial v}{\partial t} & =-u \frac{\partial v}{\partial x}-v \frac{\partial v}{\partial y}-f u-g^{\prime} \frac{\partial \eta}{\partial y}+K_{\mathbf{w}} \Delta v \\
\frac{\partial \eta}{\partial t} & =-\frac{\partial(u \eta)}{\partial x}-\frac{\partial(v \eta)}{\partial y}-h_{m}\left(\frac{\partial u}{\partial x}+\frac{\partial v}{\partial y}\right) \\
\frac{\partial T_{M}}{\partial t} & =-u \frac{\partial T_{M}}{\partial x}-v \frac{\partial T_{M}}{\partial y}
\end{aligned}
$$

with $\eta$ the thickness anomaly $\eta=h-h_{m}, h_{m}$ the average value of $h, f$ the Coriolis parameter, $K_{\mathbf{w}}$ the viscosity and $g^{\prime}=g\left(\rho_{0}-\rho_{1}\right) / \rho_{0}$ the reduced gravity. $\rho_{0}$ corresponds to the reference density and $\rho_{1}$ to the average density of the mixed layer.

As explained in the introduction, we propose to group all geophysical forces that do not correspond to advection in a unique term, that corresponds to the acceleration and is denoted by $\mathbf{a}$. The variable $\eta$ is then considered as an hidden variable of the system. In such way, System (2, 3, 4) reduces to:

$$
\begin{aligned}
& \frac{\partial u}{\partial t}=-u \frac{\partial u}{\partial x}-v \frac{\partial u}{\partial y}+a_{u} \\
& \frac{\partial v}{\partial t}=-u \frac{\partial v}{\partial x}-v \frac{\partial v}{\partial y}+a_{v}
\end{aligned}
$$

where $\mathbf{a}=\left(a_{u} a_{v}\right)^{T}$ expresses the discrepancy to the Lagrangian constancy of velocity:

$$
\frac{d \mathbf{w}}{d t}=\frac{\partial \mathbf{w}}{\partial t}+(\mathbf{w} \cdot \nabla) \mathbf{w}=\mathbf{a}
$$

From Equations (2) and (3), we get:

$$
\begin{aligned}
& a_{u}=f v-g^{\prime} \frac{\partial \eta}{\partial x}+K_{\mathbf{w}} \Delta u \\
& a_{v}=-f u-g^{\prime} \frac{\partial \eta}{\partial y}+K_{\mathbf{w}} \Delta v
\end{aligned}
$$

where $\eta$ verifies Eq. (44).

Our approach estimates $\mathbf{w}(0)$ and the acceleration term $\mathbf{a}(t)$ at each date $t \in[0, \mathrm{~T}]$, thanks to the data assimilation process summarised in Section 3 . Deriving the values $\mathbf{a}(t)$ permits describing empirically the physical processes generating the image sequence. 
Eqs. (8) and (5) are further contracted in an evolution model $\mathbf{M}$ of the state vector $\mathbf{X}$ :

$$
\frac{\partial \mathbf{X}}{\partial t}+\mathbb{M}(\mathbf{X})=\left(\begin{array}{l}
\mathbf{a} \\
0
\end{array}\right)
$$

An observation equation links the state vector to the observed Sea Surface Temperature images acquisitions $T$ :

$$
\mathbb{H} \mathbf{X}=T+\varepsilon_{R}
$$

The observation operator $\mathbb{H}$ projects the state vector into the space of image observations and consequently: $\mathbb{H X}=T_{M}$. The term $\varepsilon_{R}(\mathbf{x}, t)$ models the acquisition noise and the uncertainty on the state vector value. This last comes from the approximation of the model and from the discretization errors.

Some approximate knowledge of the value $\mathbf{X}(0)$ could be available and named background $\mathbf{X}_{b}$. However, the result of the state vector at date 0 is not exactly equal to that background value and a term $\varepsilon_{B}$ is therefore introduced:

$$
\mathbf{X}(\mathbf{x}, 0)=\mathbf{X}_{b}(\mathbf{x})+\varepsilon_{B}(\mathbf{x})
$$

The variables $\varepsilon_{R}$ and $\varepsilon_{B}$ are supposed independent, unbiased, Gaussian and characterised by their respective covariance matrices $R$ and $B$.

Eqs. 111, 12, 13) summarise the whole knowledge that is available for modelling the surface dynamics. This knowledge is processed by our approach thanks to the data assimilation algorithm that is shortly described in the next section.

\section{Data Assimilation}

In the data assimilation scientific community, an approach, named weak 4D-Var, has been defined in order to obtain the solution $\mathbf{X}$ that solves System 11,12 , 13). A cost function is first designed:

$$
\begin{aligned}
J\left[\varepsilon_{B}, \mathbf{a}\right]= & \left\langle\varepsilon_{B}, B^{-1} \varepsilon_{B}\right\rangle+\int_{t} \gamma\|\nabla \mathbf{a}(t)\|^{2} \\
& +\int_{t}\left\langle\mathbb{H} \mathbf{X}(t)-T(t), R^{-1}(\mathbb{H} \mathbf{X}(t)-T(t))\right\rangle
\end{aligned}
$$

where $\langle\cdot, \cdot\rangle$ denotes the canonical inner product in an abstract Hilbert space on which the state vector is defined, with norm $\|\cdot\|^{2}$ and $\|\nabla \mathbf{a}\|^{2}=\left\langle\nabla a_{u}, \nabla a_{u}\right\rangle+$ $\left\langle\nabla a_{v}, \nabla a_{v}\right\rangle$. The function $J$ is then minimised with control on $\varepsilon_{B}$ and on the values of the acceleration term $\mathbf{a}$.

The first term of $J$ comes from Eq. 13$)$ and expresses that the value $\mathbf{X}(0)$ at date 0 should stay close to the background value $\mathbf{X}_{b}$. It should be noted that the control on $\varepsilon_{B}$ is equivalent to the control on initial condition as $\varepsilon_{B}(\mathbf{x})=$ $\mathbf{X}(\mathbf{x}, 0)-\mathbf{X}_{b}(\mathbf{x})$. The second term constrains the acceleration term $\mathbf{a}(t)$ to be spatially smooth. The last term, coming from Eq. (12), expresses that the pseudotemperature value $T_{M}$ has to be close to that of satellite acquisitions at the end of the assimilation process. 
The gradient of $J$ is derived with the calculus of variations, as given in 9]. Its two components are:

$$
\begin{gathered}
\frac{\partial J}{\partial \varepsilon_{B}}\left[\varepsilon_{B}, \mathbf{a}\right]=2\left(B^{-1} \varepsilon_{B}+\lambda(0)\right) \\
\frac{\partial J}{\partial \mathbf{a}(t)}\left[\varepsilon_{B}, \mathbf{a}\right]=2(-\gamma \Delta \mathbf{a}(t)+\lambda(t))
\end{gathered}
$$

with $\lambda(t)$ being the adjoint variable, that is computed backward in time with the two following equations:

$$
\begin{aligned}
\lambda(\mathrm{T}) & =0 \\
-\frac{\partial \lambda}{\partial t}+\left(\frac{\partial \mathbf{M}}{\partial \mathbf{X}}\right)^{*} \lambda & =\mathbb{H}^{T} R^{-1}(\mathbb{H} \mathbf{X}-T)
\end{aligned}
$$

The adjoint operator $\left(\frac{\partial \mathbf{M}}{\partial \mathbf{X}}\right)^{*}$ verifies the following property. For all functions $\eta$ and $\lambda$ of the studied Hilbert spaces, it comes:

$$
\langle Z \eta, \lambda\rangle=\left\langle\eta, Z^{*} \lambda\right\rangle \text {. }
$$

Proof: For sake of simplicity, we suppose in this proof that Eq. (11) is written as $\frac{\partial \mathbf{X}}{\partial t}+\mathbb{M}(\mathbf{X})=\mathbf{a}$, a denoting simultaneously the acceleration involved in motion evolution and $\left(\begin{array}{l}\mathbf{a} \\ 0\end{array}\right)$.

The state vector and the functional $J$ depend on $\varepsilon_{B}$ and $\mathbf{a}(t)$. Let $\delta J$ and $\delta \mathbf{X}$ be the perturbations on $J$ and $\mathbf{X}$ obtained if $\varepsilon_{B}$ and $\mathbf{a}(t)$ are respectively perturbed by $\delta \varepsilon_{B}$ and $\delta \mathbf{a}(t)$.

From the definition of $J$, we obtain:

$$
\begin{aligned}
\frac{\delta J}{2}= & \left\langle\delta \varepsilon_{B}, B^{-1} \varepsilon_{B}\right\rangle+\int_{t} \gamma\langle\nabla \delta \mathbf{a}(t), \nabla \mathbf{a}(t)\rangle \\
& +\int_{t}\left\langle\delta \mathbf{X}(t), \mathbb{H}^{T} R^{-1}[\mathbb{H} \mathbf{X}(t)-T(t)]\right\rangle
\end{aligned}
$$

The evolution equation of $\mathbf{X}$, Eq. (11), gives:

$$
\frac{\partial \delta \mathbf{X}(t)}{\partial t}+\frac{\partial \mathbb{M}}{\partial \mathbf{X}} \delta \mathbf{X}(t)=\delta \mathbf{a}(t)
$$

and that of background, Eq. 13 :

$$
\delta \mathbf{X}(0)=\delta \varepsilon_{B}
$$

Eq. (20) gives, after multiplication by $\lambda(t)$ and integration on the space-time domain, the following equality:

$$
\int_{t}\left\langle\frac{\partial \delta \mathbf{X}(t)}{\partial t}, \lambda(t)\right\rangle+\int_{t}\left\langle\frac{\partial \mathbb{M}}{\partial \mathbf{X}} \delta \mathbf{X}(t), \lambda(t)\right\rangle=\int_{t}\langle\delta \mathbf{a}(t), \lambda(t)\rangle
$$


Integration by parts is applied on the first term and the definition of adjoint operator is used in the second one in order to obtain:

$$
\begin{array}{r}
\langle\delta \mathbf{X}(\mathrm{T}), \lambda(\mathrm{T})\rangle-\langle\delta \mathbf{X}(0), \lambda(0)\rangle-\int_{t}\left\langle\delta \mathbf{X}(t), \frac{\partial \lambda(t)}{\partial t}\right\rangle \\
+\int_{t}\left\langle\delta \mathbf{X}(t),{\frac{\partial \mathbf{M}^{*}}{\partial \mathbf{X}}}^{*}(t)\right\rangle=\int_{t}\langle\delta \mathbf{a}(t), \lambda(t)\rangle
\end{array}
$$

From Eq.17a), it comes that $\langle\delta \mathbf{X}(\mathrm{T}), \lambda(\mathrm{T})\rangle$ has a null value. From Eq. (21) it comes that $\langle\delta \mathbf{X}(0), \lambda(0)\rangle$ is equal to $\left\langle\delta \varepsilon_{B}, \lambda(0)\right\rangle$. Eq. $\left.17 \mathrm{~b}\right)$ is then used to obtain:

$$
-\left\langle\delta \mathbf{X}(t), \frac{\partial \lambda(t)}{\partial t}\right\rangle+\left\langle\delta \mathbf{X}(t), \frac{\partial \mathbb{M}^{*}}{\partial \mathbf{X}} \lambda(t)\right\rangle=\left\langle\delta \mathbf{X}(t), \mathbb{H}^{T} R^{-1}(\mathbb{H} \mathbf{X}(t)-T(t))\right\rangle
$$

and rewrite Eq. 23) as:

$$
\int_{t}\left\langle\delta \mathbf{X}(t), \mathbb{H}^{T} R^{-1}(\mathbb{H} \mathbf{X}(t)-T(t))\right\rangle=\left\langle\delta \epsilon_{B}, \lambda(0)\right\rangle+\int_{t}\langle\delta \mathbf{a}(t), \lambda(t)\rangle
$$

From this and Eq. (19), we derive:

$$
\frac{\delta J}{2}=\left\langle\delta \epsilon_{B}, B^{-1} \epsilon_{B}\right\rangle-\int_{t} \gamma\langle\delta \mathbf{a}(t), \Delta \mathbf{a}(t)\rangle+\left\langle\delta \epsilon_{B}, \lambda(0)\right\rangle+\int_{t}\langle\delta \mathbf{a}(t) \lambda(t)\rangle
$$

and obtain the gradient of $J$, as written in Eqs. (15 16).

The cost function $J$ is minimised with an iterative steepest descent method. At each iteration, the forward time integration of $\mathbf{X}$ is done, according to Eq. (11). This forward time integration provides the value of $J$. Then a backward time integration of $\lambda$, according to Eqs. (17a) and (17b), computes the value of $\nabla J$. An efficient solver, described in [120], is used to perform the optimisation given values of $J$ and $\nabla J$. To our knowledge, [7] is the first paper of the literature that describes the use of such method for estimating the initial state vector value.

\section{Numerical implementation}

Time integration of Eq. (11) relies on an explicit Euler scheme. The space discretization of motion advection, described by the two following equations:

$$
\begin{aligned}
& \frac{\partial u}{\partial t}+u \frac{\partial u}{\partial x}+v \frac{\partial u}{\partial y}=0 \\
& \frac{\partial v}{\partial t}+u \frac{\partial v}{\partial x}+v \frac{\partial v}{\partial y}=0
\end{aligned}
$$

involves a source splitting method [19], as explained below. 
Given an integration interval $\left[t_{1}, t_{2}\right]$, Eqs. 29) (where $n$ stands for non linear advection) and (30) (where $l$ stands for linear advection) are first independently integrated:

$$
\begin{array}{rrr}
\frac{\partial u^{n}}{\partial t}+u^{n} \frac{\partial u^{n}}{\partial x}=0 & t \in\left[t_{1}, t_{2}\right] \\
\frac{\partial u^{l}}{\partial t}+v \frac{\partial u^{l}}{\partial y}=0 & t \in\left[t_{1}, t_{2}\right]
\end{array}
$$

with $u^{n}\left(x, y, t_{1}\right)=u^{l}\left(x, y, t_{1}\right)=u\left(x, y, t_{1}\right)$. Then $u\left(x, y, t_{2}\right)$ is obtained as:

$$
u\left(x, y, t_{2}\right)=u^{l}\left(x, y, t_{2}\right)+u^{n}\left(x, y, t_{2}\right)-u\left(x, y, t_{1}\right)
$$

The linear advection involved in Eq. (30) is discretized by a first-order upwind scheme, as described in [4]. The nonlinear advection of Eq. 29) is first rewritten in a conservative form:

$$
\frac{\partial u}{\partial t}+\frac{\partial}{\partial x}\left(\frac{1}{2} u^{2}\right)=0
$$

and approximated by a first-order Godunov scheme 8 .

The backward time integration of the adjoint variable $\lambda$ involves the adjoint operator $\left(\frac{\partial \mathrm{M}}{\partial X}\right)^{*}$ (see Eq. $\left.17 \mathrm{~b}\right)$ ). In order to be accurate, the method requires the adjoint of the discrete model and not the discretization of the continuous adjoint. For that reason, the discrete adjoint operator $\left(\frac{\partial \mathbb{M}}{\partial X}\right)^{*}$ is derived with the automatic differentiation software Tapenade described in [3].

The background used in Eq. (13) is defined as null for motion and as the first image of the studied sequence for the pseudo-temperature. As we have no information on motion, we do not want to constrain $\mathbf{w}(0)$ to stay close to that null value. For that reason, the first term of $J$ reduces to $\left\langle\varepsilon_{B_{T}}, B_{T}^{-1} \varepsilon_{B_{T}}\right\rangle . B_{T}$ and $R$ are taken diagonal with standard deviation values corresponding to $25 \%$ of the image brightness range. $\gamma$ is given a value as small as possible: smaller values would not verify the Courant-Friedrichs-Lewy condition during the advection of the state vector.

\section{Results}

For sake of simplicity, we name in the following AM, or Acceleration Model, the model described in Sections 2 and 3 it includes the advection and the acceleration term a summarising the Coriolis, gravity and viscosity forces. We denote ME the Motion Estimation method that is obtained if suppressing $\mathbf{a}(t)$ in Eq. (11). In that case, the second term of the cost function $J$, in Eq (14), vanishes and minimisation is only controlled by $\varepsilon_{B}$.

The approach was experimented on a data base of Sea Surface Temperature sequences, acquired over Black Sea by NOAA-AVHRR sensors. The resolution of the AVHRR data is $1.1 \mathrm{~km}$ at nadir. Such image sequences are moreover able to display the dynamics of the submesoscale phenomena like eddies, jets, filaments and mushroom-like structures with a rather high temporal frequency. Our 
approach computes the surface motion fields and the trajectories of these structures by assimilating images. This high-level information is critical for improving the forecast of $3 \mathrm{D}$ ocean models such as $\mathrm{NEMO}^{5}$ when they are tuned to resolve submesoscale processes.

The approach is first validated on two synthetic experiments. Then, results on four satellite image sequences are displayed and discussed in the remaining of the section.

\subsection{Synthetic experiments}

Given initial conditions at time 0, displayed in Fig. 1. and given acceleration values $\mathbf{a}_{\text {ref }}(t)$, Eq. (11) is integrated in time and produces the ground-truth on motion and image fields. These initial conditions are similar to those on real data described in the next subsections.
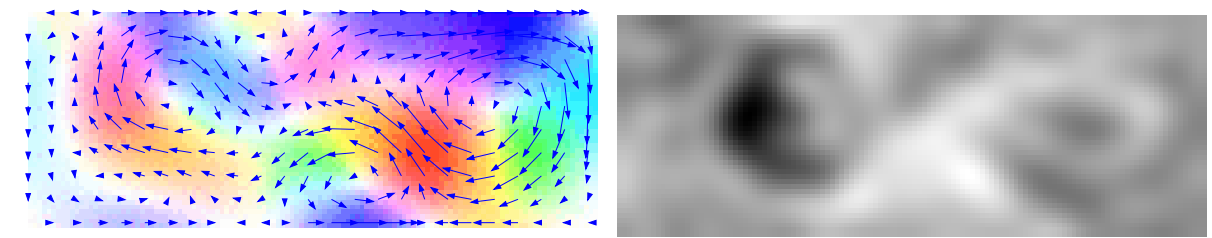

Fig. 1: Initial conditions on motion and image. The arrow representation is superposed to the coloured one.

Image snapshots are extracted from the simulation results and used for the assimilation process. The discrete assimilation window has temporal indexes from 0 to $N=84$ and observation images are available at indexes $k_{1}$ to $k_{5}=2$, $22,42,62$ and 82 .

In a first experiment, the acceleration values $\mathbf{a}_{\text {ref }}(t)$ are null. Snapshots are displayed on Fig. 2 Results are displayed on Fig. 3 for ME, AM and for the

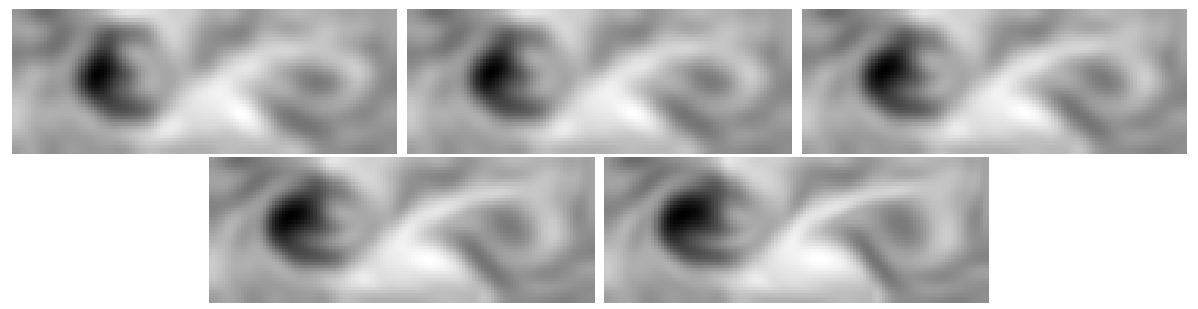

Fig. 2: Image observations.

\footnotetext{
${ }^{5}$ http://www.nemo-ocean.eu/
} 
motion estimation method of Sun et al. [13. The estimated motion and acceleration fields are respectively denoted $\mathbf{w}_{e}$ and $\mathbf{a}_{e}$. Table1 1 provides statistics on the

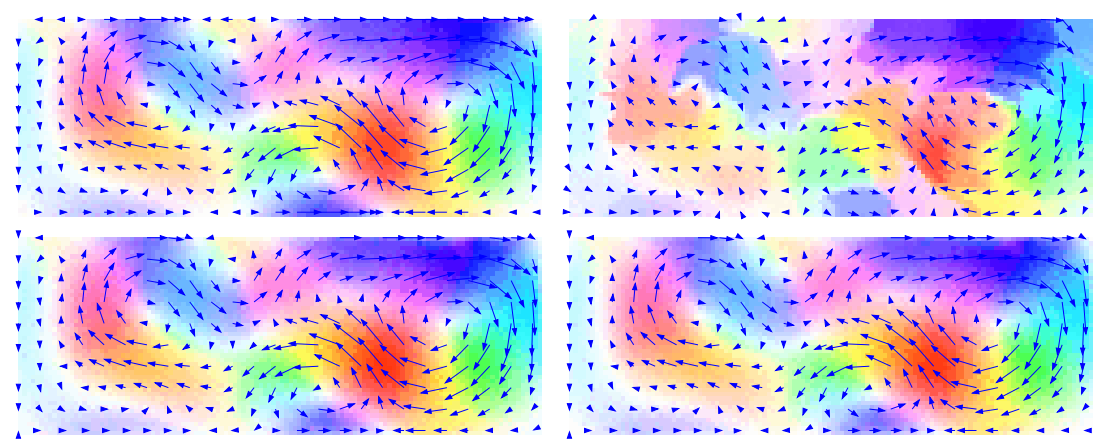

Fig. 3: First line: Groundtruth, Sun - Second line: ME, AM.

discrepancy between results and ground truth. They concern the norm $\|\mathbf{w}\|$ and orientation $\theta$ of motion. This table shows that AM estimates motion field with

\begin{tabular}{c||c||c} 
& $\left|\theta_{e}-\theta_{\text {ref }}\right|\left\|\left|\left\|\mathbf{w}_{\text {ref }}\right\|-\left\|\mathbf{w}_{e}\right\|\right| /\right\| \mathbf{w}_{\text {ref }} \|$ \\
\hline \hline method & mean & mean \\
\hline Sun & 16.1 & $43 \%$ \\
ME & 3.2 & $9 \%$ \\
AM & 3.5 & $10 \%$
\end{tabular}

Table 1: Statistics on motion errors for Sun, ME (without a) and AM (with a).

a quality comparable to ME: it demonstrates that the acceleration estimated by AM is almost null. Statistics on acceleration confirm this property as the average value of $\left\|\mathbf{a}_{e}\right\|$ is $3.6 \times 10^{-5}$ on the whole temporal interval.

In a second experiment, the acceleration $\mathbf{a}_{\text {ref }}(t)$ is null for $t \neq 41$ and equal to the vertical constant $9 \times 10^{-2}$ at $t=41$. Results are displayed on Fig. 4 Table 2 provides statistics on the discrepancy between motion results and ground-truth. Statistics on the norm of acceleration $\mathbf{a}_{e}(t)$ are displayed on Fig. 5. From Table2, it can be seen that AM gives a better estimation of velocity than Sun. As the simulation generating image data includes an acceleration, ME fails to estimate an accurate velocity. It can be seen on Fig. 5 that our approach computes an acceleration having a maximal norm value at date 41 . This means that AM correctly localises the acceleration in time. Moreover, the average orientation of $\mathbf{a}_{e}(41)$ is $90.4^{\circ}$ with a standard deviation of $11.4^{\circ}$ against $90^{\circ}$ for the ground truth. That further demonstrates the accuracy of the estimation. 


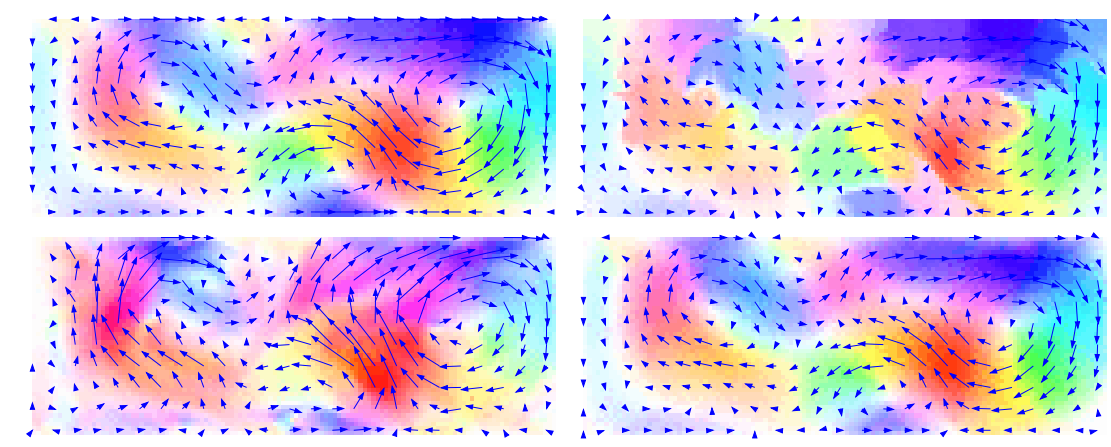

Fig. 4: First line: groundtruth, Sun - Second line: ME, AM.

\begin{tabular}{c||c||c} 
& $\left|\theta_{e}-\theta_{\text {ref }}\right||||| \mathbf{w}_{\text {ref }}\|-\| \mathbf{w}_{e}\|\mid /\| \mathbf{w}_{\text {ref }} \|$ \\
\hline \hline method & mean & mean \\
\hline Sun & 16.9 & $44 \%$ \\
ME & 36.3 & $68 \%$ \\
AM & 11.8 & $22 \%$
\end{tabular}

Table 2: Statistics on motion errors for Sun, ME (without a) and AM (with a).

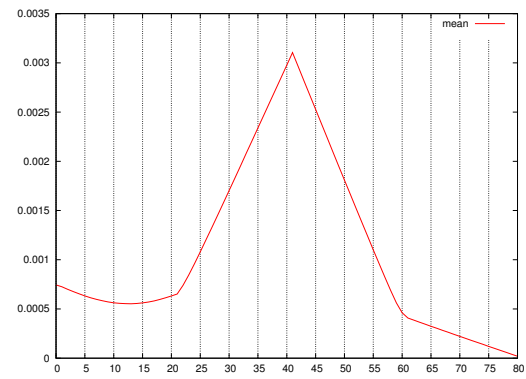

Fig. 5: Statistics on the norm of $\mathbf{a}_{e}$.

\section{$5.2 \quad$ Satellite experiments}

In a first experiment, we analyse the ability of the proposed method to correctly estimate motion, which is a natural consequence of a correct assessment of geophysical forces. For that purpose, AM results are compared with those obtained by the motion estimation method of Sun et al. 13. The satellite sequence is displayed on Fig. 6. Acquisition dates are at $30 \mathrm{~min}, 6$ hours, 15 hours, and 30 hours after the beginning of the studied temporal interval. Two gyres are clearly visible on these data. Motion results w(0), obtained by Sun and AM, are displayed on Fig. 7. AM successes to capture the two gyres while Sun's method fails. As $\mathbf{w}(0)$ is obtained from the analysis of the whole image sequence, its correct estimation means that the physical processes involved in $\mathbf{a}(t)$ are correctly 

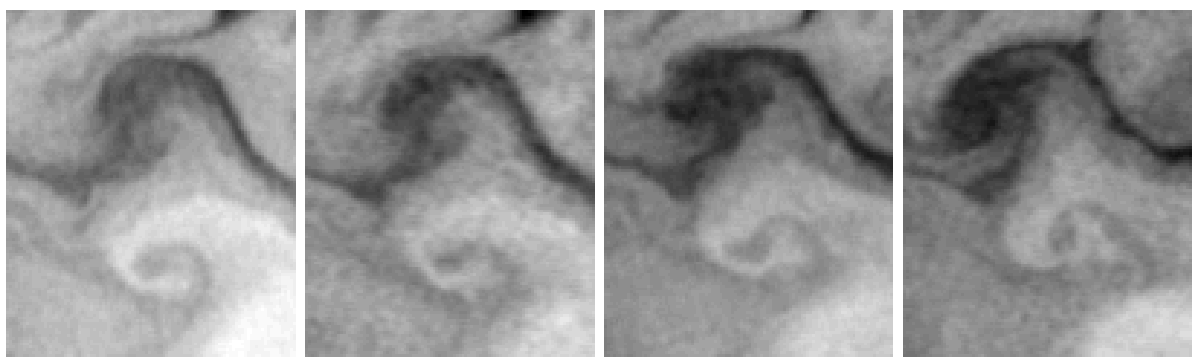

Fig. 6: Left to right: SST images acquired on October $19^{\text {th }} 2007$ over Black Sea.

assessed by the model. It also means that the non advective geophysical forces may be correctly described with the unique term $\mathbf{a}(t)$.
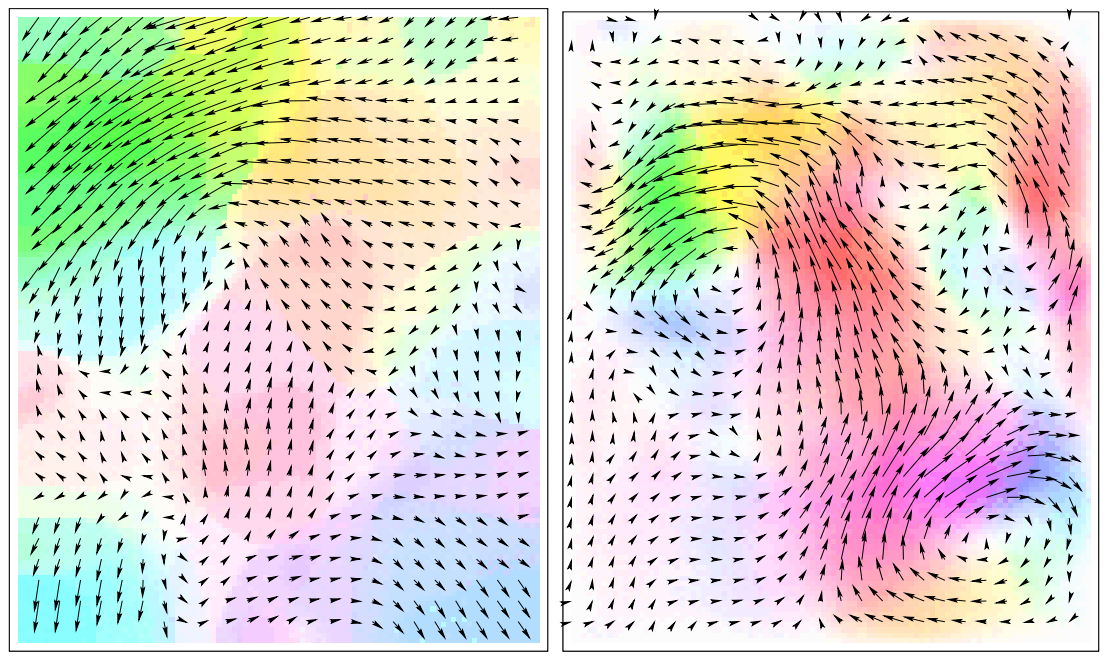

Fig. 7: Motion results computed by Sun (left) and AM (right) at first observation date.

In a second experiment, the capability of AM to track features or points of interest on the whole image sequence is examined. If the method provides accurate tracking results, this means that motion is correctly estimated on the studied temporal interval and properly transports the observed structures. A sequence of four SST images acquired on October $8^{\text {th }} 2005$ is displayed on Fig. 8. They are acquired $30 \mathrm{~min}, 10$ hours $15 \mathrm{~min}, 12$ hours and 15 hours $30 \mathrm{~min}$ after the beginning of the studied interval. Nine characteristic points are defined in white on the first observation. Points are additionally surrounded by a coloured circle that helps to discriminate them on the following observations. These points are considered as characteristic, because they sample the various types of trajectories that can be observed on the sequence. On observations 2 to 4 , the position 
of these nine points obtained with Sun's method are displayed in red while those obtained with AM are in blue. On the fourth acquisition, in the "light pink circle" on the upper right, the point obtained with Sun's method is outside of the image domain. Looking at the trajectories, it can be observed that Sun's algorithm fails to track these characteristic points, due to a wrong estimation of motion.

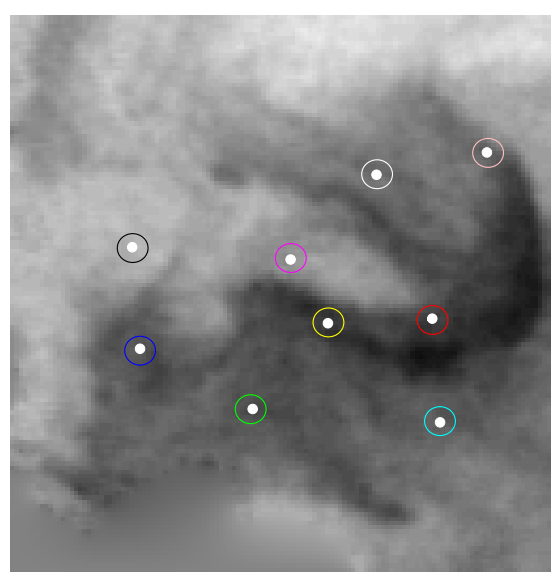

(a) Observation 1

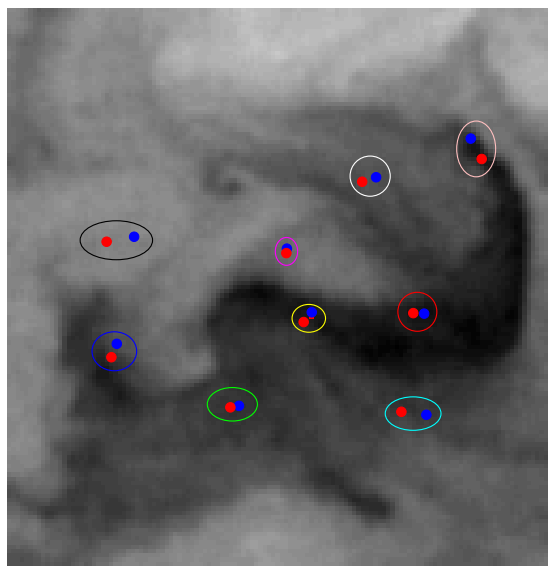

(c) Observation 3

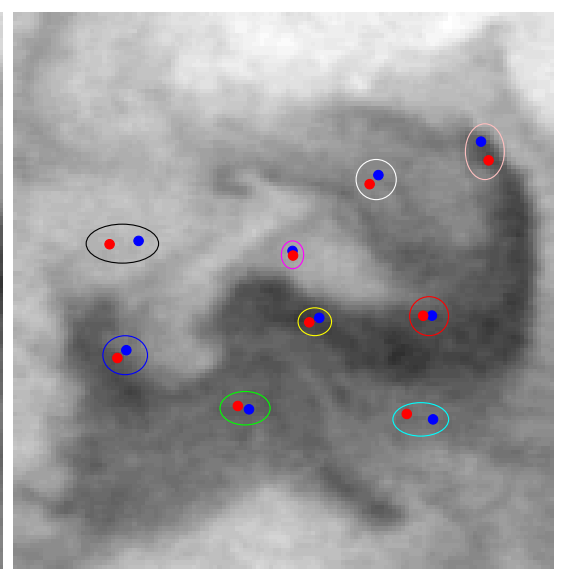

(b) Observation 2

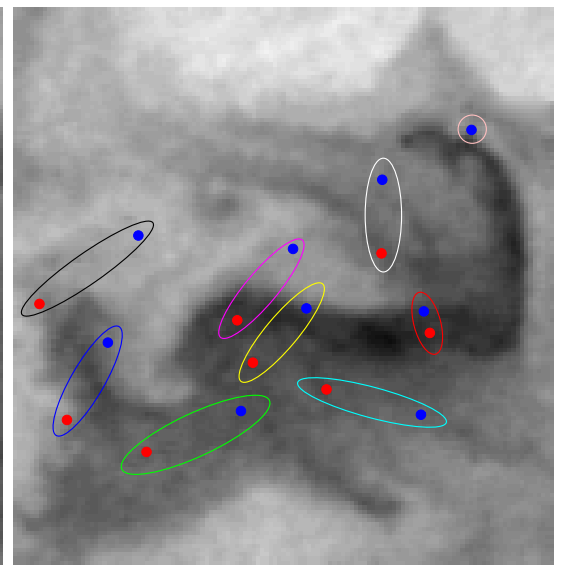

(d) Observation 4

Fig. 8: Tracking of characteristic points. Results of Sun correspond to red points, those of AM to blue points.

Another sequence of five SST images, acquired on July $27^{\text {th }} 2007$ is displayed on Fig. 9. The sensor acquired data 30 min, 8 hours 15 min, 13 hours, 22 hours 
30 min and 24 hours 30 min after the beginning of the studied interval. Seven characteristic points are defined in white on the first observation. On the next ones, positions obtained with Sun's method are displayed in red while those obtained with AM are in blue. At the second date, two points are at the same position with Sun and AM: only the red point is visible as the blue one is hidden behind it. On the fourth observation, one red point has disappeared from the display as it is located outside of the image domain. On the last frame, the colour of the ellipse surrounding each couple of points gives an additional information on the quality of the result: a blue ellipse means that our method gives the best result while the white one means that both methods are equivalent. Again,

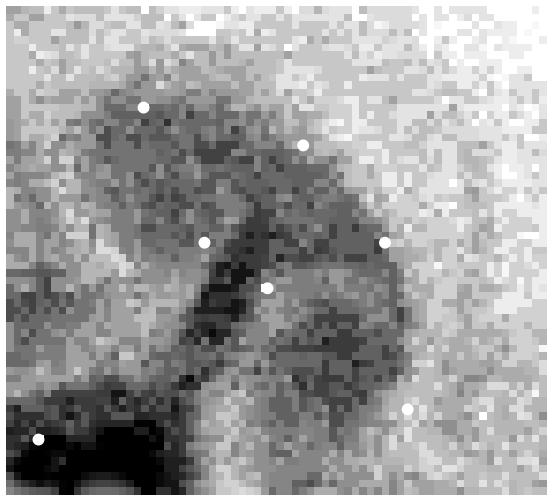

(a) Observation 1

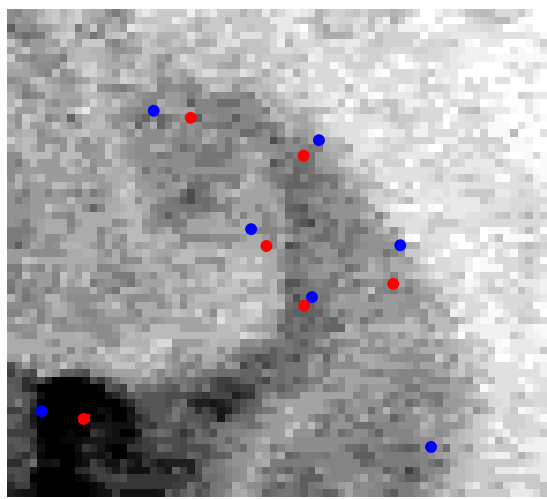

(c) Observation 4

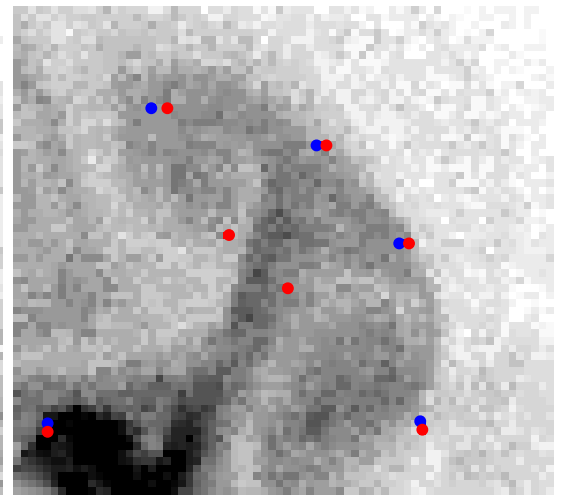

(b) Observation 2

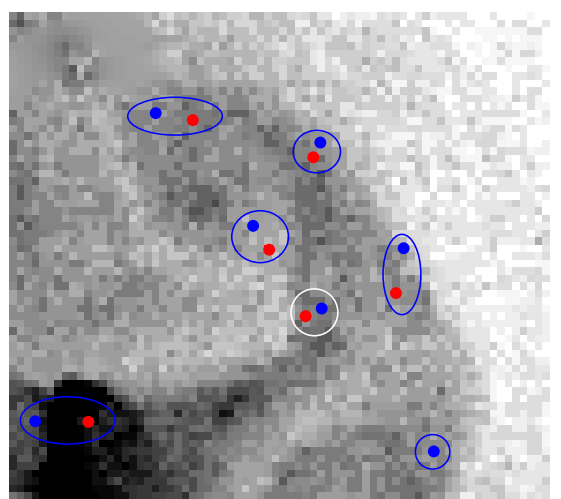

(d) Observation 5

Fig. 9: Tracking of characteristic points. A blue ellipse on (d) expresses that AM is the best while the white ellipse expresses that results are equivalent.

Sun's motion results fail to track characteristic points on these data as physical processes are not correctly assessed by the underlying image model. 
Last, AM is also compared with the optical flow estimation of Suter [14, that is dedicated to fluid flows, on a sequence of five images acquired on May $14^{\text {th }} 2005$. The acquisitions were obtained $30 \mathrm{~min}, 2$ hours and $45 \mathrm{~min}, 5$ hours and $15 \mathrm{~min}, 7$ hours and $15 \mathrm{~min}$, and 16 hours and 15 min after the beginning of the studied interval. As previously, six feature points are chosen on the first observation and displayed on the upper image of Fig. 10. Their final position on the fifth observation is given in the lower part of the same figure. Suter's and

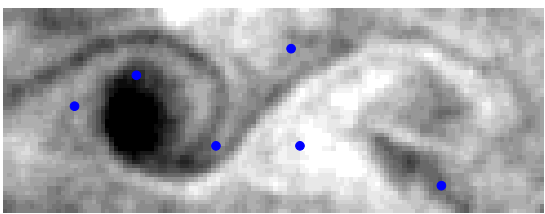

(a) Characteristic points on the first observation

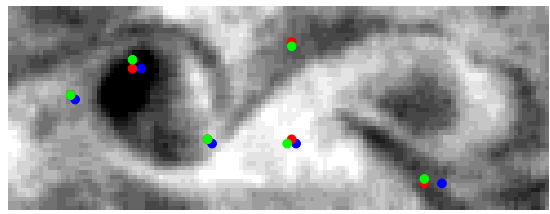

(c) Observation 3

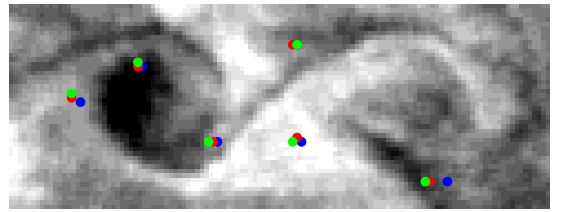

(b) Observation 2

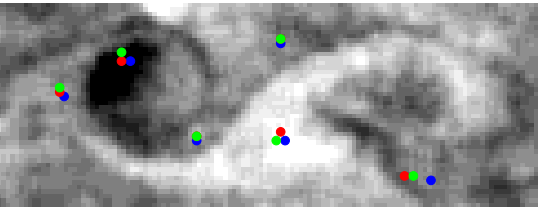

(d) Observation 4

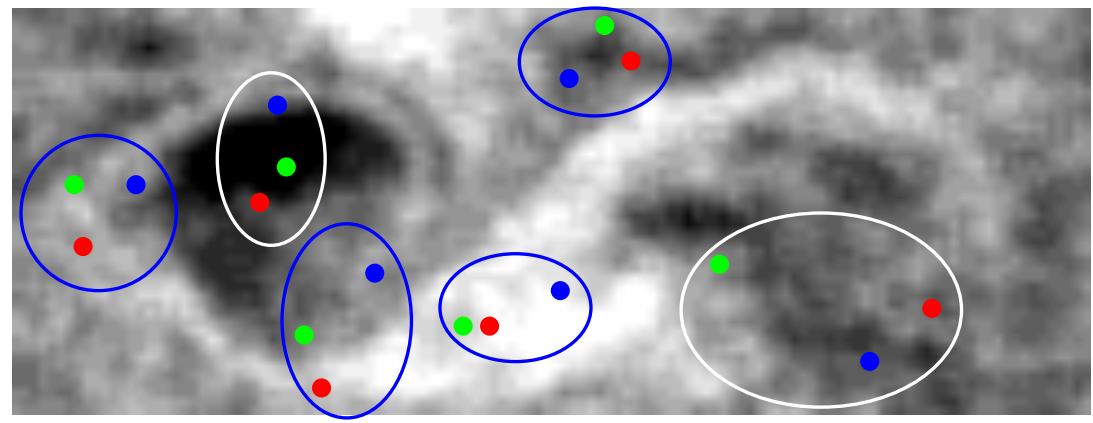

(e) Observation 5 .

Fig. 10: Sun: red, Suter: green, AM: blue.

Sun's methods are both only constrained by grey level values and do not rely on the underlying dynamics. However, Suter's algorithm provides better result than Sun's method, because it is specifically designed for fluid flows motion. In particular, it correctly assesses rotational motion. From left to right in Subfigure 10(e): AM gives the best result for the first, third, fourth and fifth points (blue ellipses). For the second and sixth (white ellipses) points, it is not possible 
to determine which one from Suter and AM provides the best result. The same conclusion is valid for all studied image sequences.

\section{Conclusion}

This paper describes how to learn the ocean surface dynamics from an image model, AM, that summarises the shallow water equations by an advection term and an acceleration term a. This last represents physical processes such as the Coriolis force, the gravity force and the viscosity. A data assimilation algorithm was designed for AM that estimates the velocity field at the first acquisition date and the acceleration $\mathbf{a}(t)$ at each date of the studied interval. The function $\mathbf{a}(t)$ is of major importance for correctly assessing the hidden physical processes and accurately estimating motion on the whole image sequence. The method has been quantified on synthetic data and illustrated on several SST sequences of Black Sea. On these last experiments, a display was given on motion fields and on the tracking of characteristic points. Moreover, the approach was compared with state-of-the-art optical flow algorithms. The conclusion is that modelling the acceleration, even as a simple unique term, improves motion estimation and allows tracking of structures.

The short-term perspectives of this research work will be to compare the acceleration term $\mathbf{a}(t)$ with forces involved in the shallow water model, in order to further validate the ability of the empirical model to assess geophysical processes.

\section{Acknowledgements}

Data have been provided by E. Plotnikov and G. Korotaev from the Marine Hydrophysical Institute of Sevastopol, Ukraine.

\section{References}

1. Byrd, R.H., Lu, P., Nocedal, J.: A limited memory algorithm for bound constrained optimization. Journal on Scientific and Statistical Computing 16(5), 1190-1208 (1995)

2. Dee, D.: Bias and data assimilation. Quaterly Journal of the Royal Meteorological Society 131, 3323-3343 (2005)

3. Hascoët, L., Pascual, V.: The Tapenade Automatic Differentiation tool: Principles, Model, and Specification. ACM Transactions On Mathematical Software 39(3) (2013)

4. Hundsdorfer, W., Spee, E.: An efficient horizontal advection scheme for the modeling of global transport of constituents. Monthly Weather Review 123(12), 3,554$3,564(1995)$

5. Huot, E., Herlin, I., Mercier, N., Plotnikov, E.: Estimating apparent motion on satellite acquisitions with a physical dynamic model. In: International Conference on Image Processing. pp. 41-44 (Aug 2010) 
6. Korotaev, G.K., Huot, E., Le Dimet, F.X., Herlin, I., Stanichny, S.V., Solovyev, D.M., Wu, L.: Retrieving ocean surface current by 4 -D variational assimilation of sea surface temperature images. Remote Sensing of Environment 112(4), 1464-1475 (Apr 2008), special issue on data assimilation

7. Le Dimet, F., Talagrand, O.: Variational algorithms for analysis and assimilation of meteorological observations: theoretical aspects., pp. 97-110. Tellus (1986)

8. LeVeque, R.: Numerical Methods for Conservative Laws. Lectures in Mathematics, ETH Zürich, Birkhaüser Verlag, 2nd edn. (1992)

9. Lions, J.L.: Optimal Control of Systems Governed by Partial Differential Equations. Springer-Verlag (1971)

10. Oguz, T., La Violette, P., Unluata, U.: The upper layer circulation of the Black Sea: Its variability as inferred from hydrographic and satellite observations. Journal of geophysical research 78(C8), 12,569-12,584 (Aug 1992)

11. Papadakis, N., Corpetti, T., Mémin, E.: Dynamically consistent optical flow estimation. In: Proceedings of International Conference on Computer Vision. Rio de Janeiro, Brazil (Oct 2007)

12. Sasaki, Y.: Some basic formalisms in numerical varational analysis. Monthly Weather Review 98(12), 875-883 (Dec 1970)

13. Sun, D., Roth, S., Black, M.: Secrets of optical flow estimation and their principles. In: Proceedings of European Conference on Computer Vision. pp. 2432-2439 (2010)

14. Suter, D.: Motion estimation and vector splines. In: Proceedings of Conference on Computer Vision and Pattern Recognition. pp. 939-942 (1994)

15. Titaud, O., Vidard, A., Souopgui, I., Le Dimet, F.X.: Assimilation of image sequences in numerical models. Tellus A 62, 30-47 (2010)

16. Trémolet, Y.: Accounting for an imperfect model in 4D-Var. Quaterly Journal of the Royal Meteorological Society 132(621), 2483-2504 (Oct 2006)

17. Vallis, G.K.: Atmospheric and oceanic fluid dynamics. Cambridge University Press (2006), 745 pp.

18. Valur Hólm, E.: Lectures notes on assimilation algorithms. Tech. rep., European Centre for Medium-Range Weather Forecasts Reading, U.K (Apr 2008)

19. Wolke, R., Knoth, O.: Implicit-explicit Runge-Kutta methods applied to atmospheric chemistry-transport modelling. Environmental Modelling and Software 15, 711-719 (Sep 2000)

20. Zhu, C., Byrd, R., Lu, P., Nocedal, J.: L-BFGS-B: Algorithm 778: L-BFGS-B, FORTRAN routines for large scale bound constrained optimization. ACM Transactions on Mathematical Software 23(4), 550-560 (1997) 